\title{
Strange Predictions by Binary Heterogeneous Nucleation Theory Compared with a Quantitative Experiment ${ }^{\dagger}$
}

\author{
M. Kulmala,*,» A. Lauri, ${ }^{\ddagger}$ H. Vehkamäki,, A. Laaksonen, ${ }^{\S}$ D. Petersen," and P. E. Wagner \\ Department of Physics, University of Helsinki, P.O. Box 64, FIN-00014 University of Helsinki, Finland, \\ Department of Applied Physics, University of Kuopio, P.O. Box 1627, FIN-70211 Kuopio, Finland, and Institut \\ für Experimentalphysik, Universität Wien, Boltzmanngasse 5, A-1090 Wien, Austria
}

Received: May 8, 2001; In Final Form: August 28, 2001

\begin{abstract}
Binary heterogeneous nucleation of water- $n$-propanol and water-sulfuric acid mixtures has been investigated. The classical thermodynamically consistent theory has been used. In the case of the water- $n$-propanol system, physically unrealistic predictions are seen. At water-gas-phase activities above 1, the theory predicts negative molecular occupation numbers of water in the critical clusters. In the case of water-sulfuric acid, however, the predictions are realistic. A series of quantitative experiments of heterogeneous nucleation of water $-n$ propanol on oxidized silver particles have been performed, and the results are contrasted with the theoretical predictions.
\end{abstract}

\section{Introduction}

Formation of atmospheric aerosols has recently received growing experimental and theoretical interest due to climate and health related effects of fine particles. ${ }^{1,2}$ Although new theories have been developed (see, e.g., Laaksonen et al. $(1995)^{3}$ ), including molecular dynamics (e.g., Arstila et al. (1998) ${ }^{4}$ ) and Monte Carlo simulations, ${ }^{5}$ the classical nucleation theory (see, e.g., Seinfeld and Pandis $(1998)^{6}$ ) is the only one, which is practical for atmospheric applications, and particularly in atmospheric models. The classical nucleation theory requires as input detailed representations of various thermodynamic parameters. ${ }^{7,8}$ However, laboratory experiments and also molecular approaches are needed to confirm the results obtained by classical theories, and in the future, parametrized versions of molecular models can hopefully be used in atmospheric models.

The classical theory of binary homogeneous nucleation was first treated in the 1930s by Flood (1934), ${ }^{9}$ but it was not until almost 20 years later that Reiss $(1950)^{10}$ published a complete treatment of binary nucleation. Doyle $(1961)^{11}$ was the first to publish predicted nucleation rates for the sulfuric acid-water system. Because a free sulfuric acid molecule tends to gather water molecules around it to form hydrates, Heist and Reiss $(1974)^{12}$ and Jaecker-Voirol et al. (1987) ${ }^{13}$ improved the classical theory taking into account the effect of sulfuric acid hydration. Stabilizing the vapor, hydrates reduce nucleation rates by a factor of $10^{3}-10^{8}$. Wilemski $(1984)^{14}$ presented a revised classical nucleation theory, and pointed out that the previous standard nucleation theory (Doyle, 1961) ${ }^{11}$ was thermodynamically inconsistent: the numerical method to search the critical cluster (a smallest thermodynamically stable cluster) was not correct, and it resulted in wrong cluster compositions and nucleation rates (Laaksonen et al. (1993) $)^{15}$ ). Kulmala et al. (1998) ${ }^{16}$ have performed parametrizations for the nucleation rate as a function

\footnotetext{
$\dagger$ Part of the special issue "Howard Reiss Festschrift".

$\doteqdot$ Department of Physics.

$\S$ Department of Applied Physics.

"Institut für Experimentalphysik.
}

of temperature, relative humidity, and acidity using thermodynamically correct theory.

Condensational growth of insoluble aerosol particles is often initiated by heterogeneous nucleation on the surface of these particles. The classical theory of heterogeneous nucleation was developed by Fletcher (1958). ${ }^{17}$ The theory was extended to binary systems using the capillarity approximation by Lazaridis et al. (1991). ${ }^{18}$ Heterogeneous nucleation on insoluble particles initiates changes in particle size and composition distributions, but does not increase particle number concentration. Soluble aerosol particles may grow as a result of equilibrium uptake of vapors (mostly water), but only when the vapor becomes supersaturated significant mass transfer in the form of condensation can take place between the phases.

The quantification of heterogeneous nucleation is even more difficult than that of homogeneous nucleation. This is due to the complexity of interactions between the nucleating molecules and the underlying surface. Heterogeneous nucleation rate is strongly dependent on the characteristics of the surface, and it is extremely difficult to produce well-defined surfaces for experimental investigations. The lack of experimental data, on the other hand, has made it difficult so far to verify any theoretical ideas. In the present paper we report measurements of nucleation probabilities and onset activities for binary heterogeneous nucleation on monodispersed Ag particles under well-defined conditions. Quantitative comparison with corresponding model calculations provides information on the applicability of heterogeneous nucleation theory. It seems probable that in the future further information on the details of heterogeneous nucleation phenomena will be acquired through molecular dynamics or Monte Carlo simulations. ${ }^{19}$

A further complication emerges when one tries to carry out calculations of heterogeneous nucleation at ambient conditions: in the lab, at least the surface materials are known, but this is usually not the case in the atmosphere. The uncertainties associated with atmospheric heterogeneous nucleation calculations can therefore be very large. However, some guidance may be acquired about the conditions in which heterogeneous nucleation can take place using the classical nucleation theory, 
which will be reviewed below (section 2). The quantitative experiments are described in section 3 , the modeling results and a comparison to the experimentally obtained data are discussed in section 4.

\section{Theory}

In this work the extended classical theory of binary heterogeneous nucleation was applied to water- $n$-propanol and water-sulfuric acid mixtures. In this theory, the critical embryos, although sometimes consisting only of a few molecules, are considered to be objects with macroscopic properties. The physicochemical properties needed for the calculations are surface tension, contact angle, density, saturation vapor pressures, and activities as a function of composition and temperature. The effect of hydrate formation in the sulfuric acidwater mixture is taken into account using the classical hydrate interaction model. ${ }^{20}$

2.1. Energy Barrier. First we summarize the theory for homogeneous nucleation. Consider a binary cluster consisting of $n_{\mathrm{w}}$ molecules of species $\mathrm{w}$ and $n_{\mathrm{a}}$ molecules of species $a$ suspended in supersaturated vapor where the temperature is $T$, vapor pressure $P_{\mathrm{v}}$, and the mole fraction of species a in the vapor is $x_{\mathrm{av}}$. The cluster is modeled as a uniform spherical liquid droplet of radius $r$ and volume $V=4 / 3 \pi r^{3}$. Let $\rho_{\text {il }}$ be the number density of species $i$ in the uniform liquid. The total number of molecules of species $\mathrm{i}$ is expressed as $n_{\mathrm{i}}=n_{\mathrm{il}}+n_{\mathrm{is}}$, where the number of molecules in the bulk phase is $n_{\mathrm{il}}=\rho_{\mathrm{il}} V$, and $n_{\mathrm{is}}$ is the surface excess number of molecules that corrects for the difference between the density profiles of our uniform droplet model and the actual cluster. The critical cluster size (denoted by the asterisk) can be found by minimizing the formation free energy of a cluster with respect to $n_{\mathrm{a}}$ and $n_{\mathrm{w}}$ (see Laaksonen et al. $(1999)^{21}$ for details). If we assume that the liquid is incompressible, we obtain the equation

$$
v_{\mathrm{a}} \Delta \mu_{\mathrm{w}}=v_{\mathrm{w}} \Delta \mu_{\mathrm{a}}
$$

which can be used to find the composition of the critical nucleus $x_{\mathrm{il}}$. Here $\Delta \mu_{\mathrm{i}}=\mu_{\mathrm{il}}\left(T, P_{\mathrm{v}}, x_{\mathrm{il}}\right)-\mu_{\mathrm{iv}}\left(T, P_{\mathrm{v}}, x_{\mathrm{iv}}\right)$ with $\mu_{\mathrm{il}}$ and $\mu_{\mathrm{il}}$ denoting the liquid- and vapor-phase chemical potentials, respectively, and $v_{\mathrm{il}}\left(T, x_{\mathrm{il}}\right)$ is the partial molecular volume of species i.

Now we fix the position of the dividing surface determined by $r^{*}$ so that it coincides with the surface of tension, which means that we require $\left[\partial \sigma / \partial r^{*}\right]=0$ to hold. ${ }^{22}$ The radius of the cluster can then be obtained from the Kelvin equations

$$
\Delta \mu_{\mathrm{i}}+\frac{2 \sigma v_{\mathrm{i}}}{r^{*}}=0 \quad(\mathrm{i}=\mathrm{w}, \mathrm{a})
$$

The free energy of formation of the critical cluster is now given by

$$
\Delta G^{*}=\frac{4}{3} \pi r^{*^{2}} \sigma
$$

It has been shown ${ }^{21}$ that the surface of tension is independent of the curvature of the droplet if (and only if) the condition

$$
n_{\mathrm{ws}} v_{\mathrm{w}}+n_{\mathrm{as}} v_{\mathrm{a}}=0
$$

holds at the surface of tension. In nucleation calculations, the surface tension of a flat surface is practically always used, since no other data is available. Thus, for the theory to be internally consistent, one has to assume (albeit implicitly) that the equimolar surface specified by eq 4 coincides with the surface of tension.

If the nucleation takes place heterogeneously, i.e., is initiated by a preexisting particle, the theory is somewhat altered. The Gibbs free energy of formation of a critical cluster from a binary mixture of vapors onto a curved surface is given by the expression $^{17}$

$$
\Delta G_{\mathrm{Het}}^{*}=\frac{1}{2} \Delta G_{\mathrm{Homo}}^{*} f(m, z)
$$

Here

$$
\begin{aligned}
f(m, z)= & 1+\left(\frac{1-m z}{g}\right)^{3}+ \\
& z^{3}\left[2-3\left(\frac{z-m}{g}\right)+\left(\frac{z-m}{g}\right)^{3}\right]+3 m z^{2}\left(\frac{z-m}{g}-1\right)
\end{aligned}
$$

with

$$
g=\left(1+z^{2}-2 m z\right)^{1 / 2}
$$

and

$$
z=\frac{R_{\mathrm{p}}}{r^{*}}
$$

where $R_{\mathrm{p}}$ is the radius of curvature of the solid surface. The contact angle $\theta$ is given by $\cos \theta=m=\left(\sigma_{13}-\sigma_{23}\right) / \sigma_{12}$, where $\sigma_{\mathrm{ij}}$ is the interfacial free energy between the phases $\mathrm{i}$ and $\mathrm{j}$. The gas phase is indexed by 1 , the cluster by 2 , and the substrate by 3 . The contact angle used in the case of water $-n$-propanol mixture was based on experiments ${ }^{23}$ yielding the following result:

$$
\theta=\frac{92.56736+388.2953 x}{1+24.12755 x}
$$

$x$ being the mole fraction in the critical cluster. Equation 9 gives the value of the contact angle in degrees. The radius $r^{*}$ of the critical cluster is the same as given by homogeneous nucleation theory. Thus, the heterogeneous critical cluster can be thought of a segment of the homogeneous nucleus specified by the contact angle and the curvature of the underlying surface.

In heterogeneous nucleation, the work of critical cluster formation is a complicated function of surface tensions, and can be presented as

$$
\begin{aligned}
& \Delta G^{*}=\frac{2 \pi\left[-3 \alpha \Delta \sigma R_{\mathrm{p}}{ }^{2}+2 R_{\mathrm{p}}{ }^{3}+\alpha^{3} \sigma_{12}{ }^{3}+\right.}{\left.\alpha \sqrt{1+\frac{R_{\mathrm{p}}\left(-2 \alpha \Delta \sigma+R_{\mathrm{p}}\right)}{\alpha^{2} \sigma_{12}{ }^{2}}} \sigma_{12}\left(\alpha \Delta \sigma R_{\mathrm{p}}-2 R_{\mathrm{p}}{ }^{2}+\alpha^{2} \sigma_{12}{ }^{2}\right)\right]}
\end{aligned}
$$

where $\Delta \sigma=\sigma_{13}-\sigma_{23}$, and $\alpha=2 v_{\mathrm{i}} / \Delta \mu_{\mathrm{i}}$. Equation 1 tells us that $\alpha$ has the same value independently of the choice of component i.

2.2. Nucleation Rate and Nucleation Probability. The nucleation rate can be expressed as ${ }^{24,25}$

$$
I=R_{\mathrm{av}} F Z \exp \left(-\frac{\Delta G^{*}}{k T}\right)
$$


Here $R_{\text {av }}$ denotes the average condensation rate and $F$ denotes the total number of nucleating molecules, clusters, particles, etc., depending on the system in question. (For instance, in homogeneous nucleation $F$ would be the total number of molecules in the vapor, and in ion-induced nucleation the number of ions.) In the case of heterogeneous nucleation the identification of $F$ is not straightforward. Several different expressions for the factor $F$ in heterogeneous nucleation rate can be found in the literature.

When very small solid particles act as condensation nuclei, the nucleation rate can be expressed as ${ }^{26}$

$$
I_{1}=R_{\mathrm{av}} N_{\mathrm{par}} Z \exp \left(-\frac{\Delta G^{*}}{k T}\right)
$$

Here $N_{\text {par }}$ is the number concentration of the solid particles.

Another formula includes the adsorption mechanism through the quantity $N^{\text {ads }}$ (the total number of molecules adsorbed per unit area on the solid nuclei). In the case of atmospheric $\mathrm{H}_{2} \mathrm{O}-$ $\mathrm{H}_{2} \mathrm{SO}_{4}$ mixture, the number of acid molecules is several orders of magnitude smaller than the number of water molecules, and it is sufficient to count only the adsorbed water molecules $N_{\mathrm{w}}^{\mathrm{ads}}$ $=\beta_{\mathrm{w}} \tau_{\mathrm{w}}$. Here $\beta_{\mathrm{i}}$ is the impinging rate of molecules of species $i$ on the surface of the solid particle and $\tau_{\mathrm{i}}$ is the time which a molecule $i$ spends on the surface of the solid particle. In the case of $n$-propanol - water mixture both water and $n$-propanol molecules have to be taken into account. Thus the total number of adsorbed molecules is $N^{\text {ads }}=N_{\mathrm{w}}^{\mathrm{ads}}+N_{\mathrm{p}}^{\mathrm{ads}}=\beta_{\mathrm{w}} \tau_{\mathrm{w}}+\beta_{\mathrm{p}} \tau_{\mathrm{p}}$.

The residence time given by $\tau=\tau_{\mathrm{o}} \exp (E / R T)$, where $\tau_{\mathrm{o}}$ is a characteristic time and $E$ is the heat of adsorption. For $E$, Lazaridis et al. (1991) ${ }^{18}$ used the latent heat of condensation given by Adamson (1982). ${ }^{27}$ Hamill et al. (1982) ${ }^{26}$ used the value $2.4 \times 10^{-16} \mathrm{~s}$ for $\tau_{0}$, and the value $10800 \mathrm{cal} / \mathrm{mol}$ for $E$, while Lazaridis et al. (1991) ${ }^{18}$ on the other handmade use of the fact that $\tau_{\mathrm{o}}$ corresponds to $1 / v_{\mathrm{o}},{ }^{27}$ where $\nu_{\mathrm{o}}$ is the characteristic frequency of vibration. The vibration between two molecules can be calculated using the nearest-neighbor harmonic oscillator approximation. The angular frequency $(\omega)$ of the oscillator is

$$
\omega=2 \pi v=\sqrt{\frac{\mathrm{d}^{2} V}{\mathrm{~d} r^{2}} \cdot \frac{1}{m_{\mu}}}
$$

where $m_{\mu}$ is the reduced mass of the two molecules. For $V$, Lazaridis et al. $(1991)^{18}$ used the modified Lennard-Jones potential of polar molecules resulting in $\tau_{\mathrm{o}}=2.55 \times 10^{-13} \mathrm{~s}$, which corresponds to the water-water interaction. For the $n$-propanol-n-propanol interaction, the calculated value is $\tau_{0}$ $=1.13 \times 10^{-12} \mathrm{~s}$. In this study the temperature-dependent value of $E$ was $10640 \mathrm{cal} / \mathrm{mol}$ for water and $11740 \mathrm{cal} / \mathrm{mol}$ for $n$-propanol at $T=285 \mathrm{~K}$.

The minimum nucleation rate is obtained by assuming that heterogeneous nucleation takes place only as the entire particle is covered by critical nuclei:

$$
I_{2}=\pi r^{* 2} N^{\mathrm{ads}} R_{\mathrm{av}} Z N_{\mathrm{par}} \exp \left(-\frac{\Delta G^{*}}{k T}\right)
$$

The maximum nucleation rate corresponds to the rate of formation of a single critical cluster on the particle:

$$
I_{3}=4 \pi R_{\mathrm{p}}^{2} N^{\mathrm{ads}} R_{\mathrm{av}} Z N_{\mathrm{par}} \exp \left(-\frac{\Delta G^{*}}{k T}\right)
$$

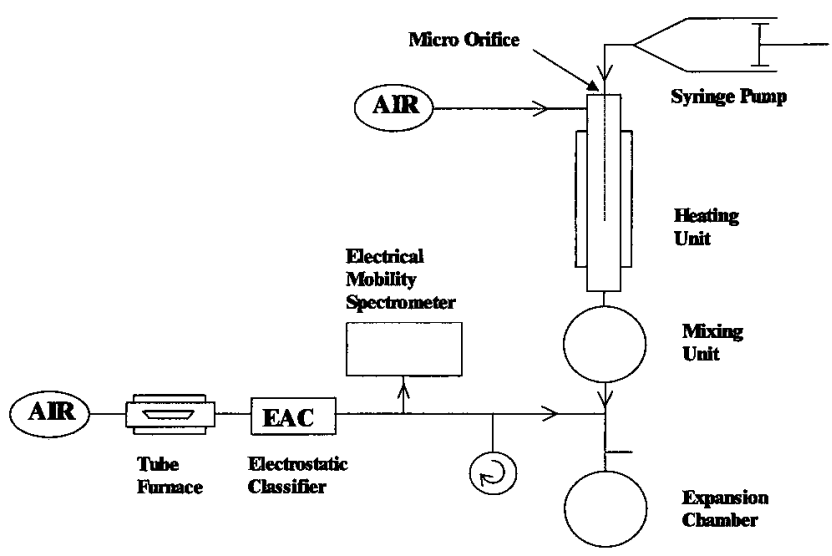

Figure 1. Schematic diagram of the experimental arrangement.

The average condensation rate $R_{\mathrm{av}}$ is given by

$$
R_{\mathrm{av}}=\frac{A \beta_{\mathrm{w}} \beta_{\mathrm{a}}}{\beta_{\mathrm{w}} \sin ^{2} \theta+\beta_{\mathrm{a}} \cos ^{2} \theta}
$$

where $A$ denotes the surface area of the embryo lying on the surface of a preexisting particle (see, e.g., Lazaridis et al. $\left.(1991)^{18}\right)$ and $\theta=\arctan [x /(1-x)]$, and $x$ is the mole fraction of species a in the nucleus. ${ }^{25}$

The effect of sulfuric acid hydration can be included in the expressions for the nucleation rate. This changes the expression for the average condensation rate slightly, but affects the work of formation of a critical cluster significantly. ${ }^{28}$

The probability for one particle to nucleate within some nucleation time $t$ is (see, e.g., Lazaridis et al. $(1992)^{28}$ )

$$
P=1-\exp \left(-I_{\mathrm{Het}} t\right)
$$

where $I_{\mathrm{Het}}$ corresponds to the maximum nucleation rate calculated above. The theoretical onset activities, where half of the number of aerosol particles are activated to growth, were determined in the model runs by setting the nucleation probability to 0.5 .

\section{Experiments}

We have performed an experimental study of heterogeneous nucleation of supersaturated binary $n$-propanol-water vapor mixtures on monodispersed Ag particles under well defined thermodynamic conditions. For the vapor compounds selected, all physicochemical parameters required are known with sufficient accuracy (see Appendix). Contact angles with respect to a Ag surface were newly measured for liquid mixtures with various mixing ratios ${ }^{23}$ using the Wilhelmy plate method and a goniometer.

A schematic diagram of the experimental arrangement is shown in Figure 1. Ag particles were generated in a tube furnace at a temperature of $1040{ }^{\circ} \mathrm{C}$. Carefully filtered and dried air was passed through the furnace tube at the constant flow rate of $1.8 \mathrm{l} / \mathrm{min}$. Ag was evaporating from a ceramic boat and subsequent cooling resulted in homogeneous nucleation of $\mathrm{Ag}$ particles. Immediately after leaving the furnace tube, the Ag aerosol was diluted with filtered and dried air with a flow rate of $1.7 \mathrm{l} / \mathrm{min}$ and a polydispersed aerosol with a particle number concentration around $10^{7} \mathrm{~cm}^{-3}$ was obtained. A monodispersed fraction of the $\mathrm{Ag}$ aerosol with an average diameter of approximately $8 \mathrm{~nm}$ and a number concentration of about $10^{5}$ 
$\mathrm{cm}^{-3}$ was extracted by means of an electrostatic aerosol classifier EAC. The size distribution of this aerosol fraction was measured using an electrical mobility spectrometer.

Binary $n$-propanol-water vapor mixtures in air were obtained by means of the spray-evaporation method. A liquid mixture with the desired mixing ratio was injected by a high precision syringe pump through a micro orifice into a heating unit at selectable constant feed rates ranging from 0.098 to $0.35 \mathrm{~mL} /$ min. Depending on the feed rate, chosen microorifices with diameters of 20 or $35 \mu \mathrm{m}$ were used. The resulting liquid beam was quantitatively evaporated and mixed with carefully filtered and dried air as well as with the monodispersed Ag aerosol at the precisely controlled flow rates of 5.1 and $1.44 \mathrm{l} / \mathrm{min}$, respectively.

The aerosol obtained having a particle number concentration of typically $20 \times 10^{3} \mathrm{~cm}^{-3}$ as well as the desired $n$-propanol and water vapor phase activities was entered into a computer controlled, pressure defined expansion chamber. Fast expansion of the binary vapor mixture leads to an adiabatic temperature drop and thus to vapor supersaturation. Particles acting as condensation nuclei will grow to optically detectable sizes.

Size and number concentration of the growing drops are measured by means of the constant-angle Mie scattering (CAMS) method. ${ }^{29}$ To this end the growing particles in the expansion chamber are illuminated by a laser beam. The light flux scattered at a selectable, constant scattering angle, as well as the light flux transmitted through the expansion chamber, are monitored by appropriate sensors, which have been calibrated relative to each other. To compensate possible influences of extinction on the scattered light flux, it is normalized relative to the light flux transmitted through the expansion chamber. The normalized scattered light flux vs time curves obtained show a quite rich morphology in quantitative agreement with corresponding to theoretical light flux vs size curves calculated according to Mie theory. After establishing a unique correspondence between experimental and theoretical light scattering extrema, size and number concentration of the growing droplets can be quantitatively determined without referring to any external empirical calibration.

Before performing experiments on heterogeneous nucleation, the actual unary vapor phase activities occurring after the adiabatic expansion were verified by comparing experimental and theoretical drop growth curves. ${ }^{30}$ In fact, drop growth rate is a very sensitive indicator for vapor supersaturations.

To investigate the heterogeneous nucleation process, we performed a measurement series with stepwise increasing vapor phase activities were and determined corresponding nucleation probabilities, i.e., number concentrations of activated droplets normalized with respect to the total aerosol number concentration. Beyond certain activities a comparatively steep increase of the nucleation probability occurs. A three-dimensional presentation of the nucleation probabilities vs the vapor phase activities of both vapor components is shown in Figure 2. It is notable that all data points refer to the same nucleation temperature of $285 \mathrm{~K}$. To obtain isothermal nucleation probability curves, the vapor phase activities were varied by changing the liquid feed rate from the syringe pump rather than changing the expansion ratio.

Figure 2 shows also the onset activities at which the nucleation probability is 0.5 . A quantitative comparison of the experimentally obtained onset activities with corresponding to theoretical calculations will be presented and discussed in the next section.
$8 \mathrm{~nm}$ oxidized $\mathrm{Ag}$ Particles at $285 \mathrm{~K}$

\section{Normalized Part. Num. Conc. [-]}

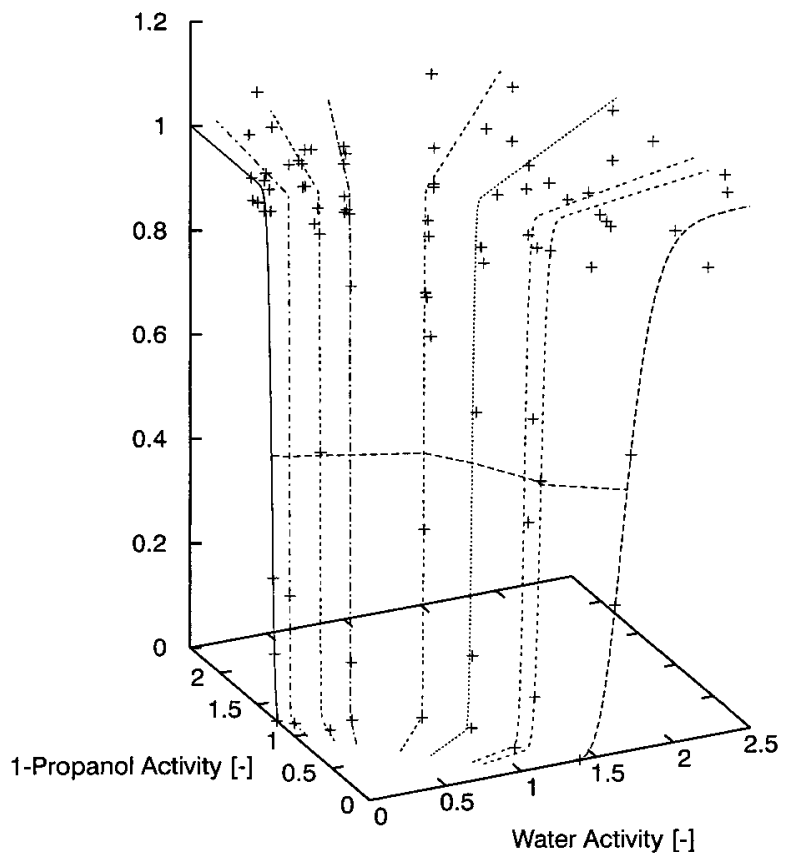

Figure 2. Experimental nucleation probabilities for Ag particles (oxidized) shown vs the vapor phase activities of water and $n$-propanol at $285 \mathrm{~K}$. The curve corresponding to the onset activities is indicated.

\section{Results and Discussion}

We have calculated the nucleation rates and nucleation probabilities on aerosol particles. Three different log-normal aerosol size distributions of mean diameters 9, 90, and $900 \mathrm{~nm}$ and standard deviation of $\sigma_{\mathrm{u}}=1.1$ were used. The nucleation time was $1 \mathrm{~ms}$. The nucleation rate and the nucleation probability depend strongly on surface tension and contact angle. In Figure 3 the surface tension between the gas and liquid phase is shown as a function of liquid composition at $T=285 \mathrm{~K}$ for both mixtures studied. The fact that $n$-propanol is very surface active is seen in Figure $3 \mathrm{~b}$ as a clear drop in the surface tension when mole fraction of $n$-propanol is increased.

The onset activities for binary heterogeneous nucleation can be obtained from nucleation probabilities. The onset activities have been calculated for both systems at different temperatures using different contact angles. According to measurements, ${ }^{23,31}$ the contact angle changes as a function of the critical cluster composition, but we have also performed calculations with various constant contact angles.

Figure $4 \mathrm{a}$ shows the modeled onset activities for water $-n$ propanol system at different temperatures, and Figure $4 \mathrm{~b}$ for different size distributions of the preexisting aerosol. The contact angle is composition dependent. A strange "hump" as well as a smaller "spike" (at water activity approximately equal to 1) can be seen clearly in the model results assuming a changing contact angle. This kind of behavior is not observed in the experiments as will be discussed below. The behavior is enhanced at lower temperatures, being almost negligible at 333 $\mathrm{K}$. We also notice that the smaller the substrate the bigger the effect is: the effect is significantly smaller for $900 \mathrm{~nm}$ than for $9 \mathrm{~nm}$ particles.

In Figure 5 the modeled onset activities for water- $n$-propanol system for different values of constant contact angle are shown. 

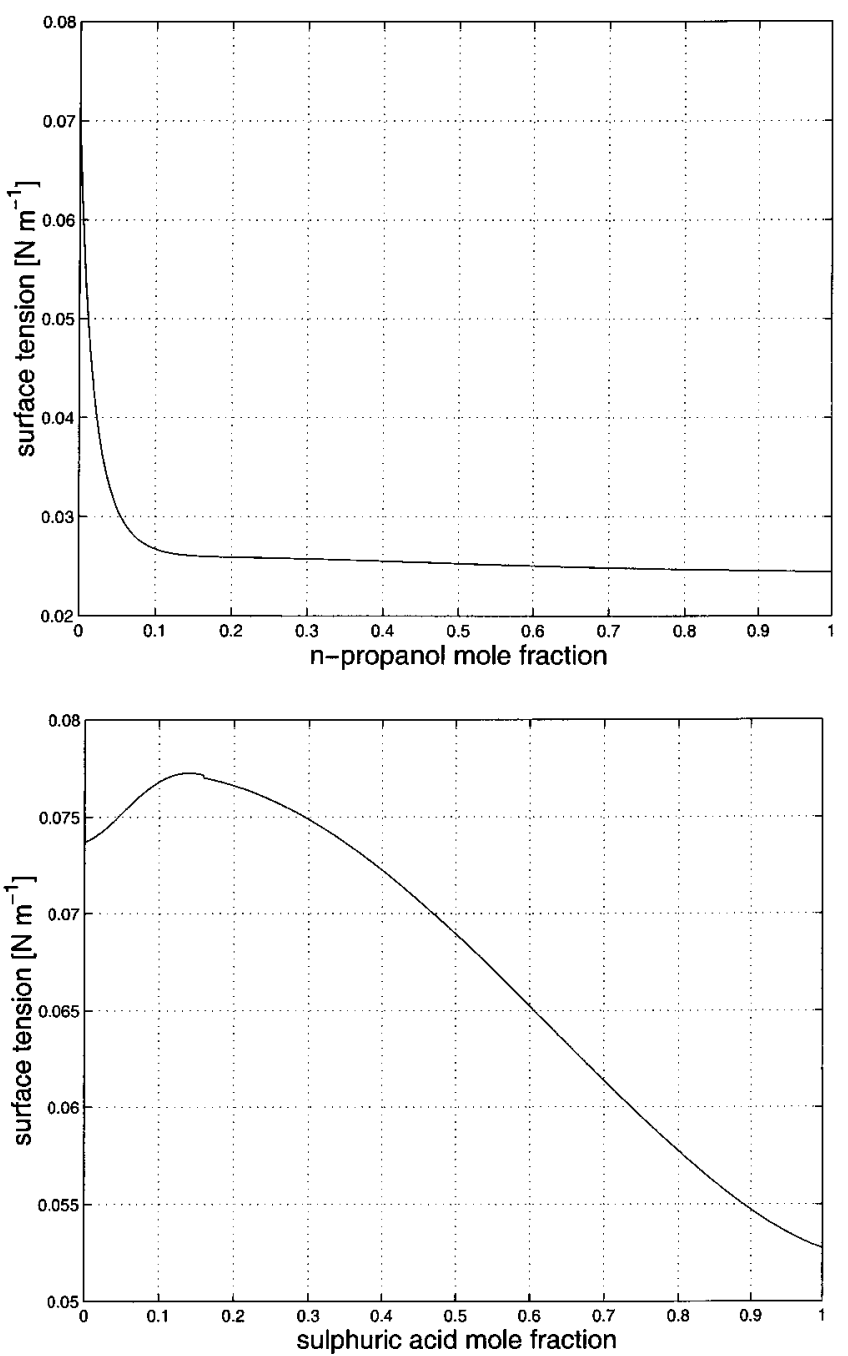

Figure 3. (a) Surface tension of water-n-propanol as a function of $n$-propanol mole fraction and (b) surface tension of water-sulfuric acid as a function of sulfuric acid mole fraction. $T=285 \mathrm{~K}$.

Both the hump and the spike disappear when the contact angle approaches zero (totally wettable surface). The odd behavior starts when the contact angle is around $30^{\circ}$. The hump is very clear when the contact angle is $90^{\circ}$ or more. The hump for homogeneous nucleation (contact angle $180^{\circ}$ ) agrees with earlier findings. ${ }^{32}$

The water-sulfuric acid system behaves differently. In this case there is no experimental data on the composition dependence of the contact angle. Thus, we are restricted to using constant contact angles. Figure 6 shows modeled onset activities for the water-sulfuric acid system for different contact angles. Onset activities do vary as a function of contact angle and temperature (Figure 7), but no strange behavior can be seen, and the onset curves are smooth.

In Figure 8 we have studied the behavior of the nucleation probability, when both the spike and the hump are clearly seen. In these figures we have used constant $n$-propanol activity (0.77) and varied the water activity between 0.8 and 4.0. We used the mole fraction dependent contact angle. The odd behavior starts when the water activity is 0.95 ; the spike can be seen as a sudden drop in the nucleation probability. The hump is seen as a sink in the nucleation probability between water activities 1.2 and 3.5. When the water activity is varied between 0.9 and 1.0 , the mole fraction in critical cluster drops steeply from 0.5 to 0.1 . (see Figure 9).
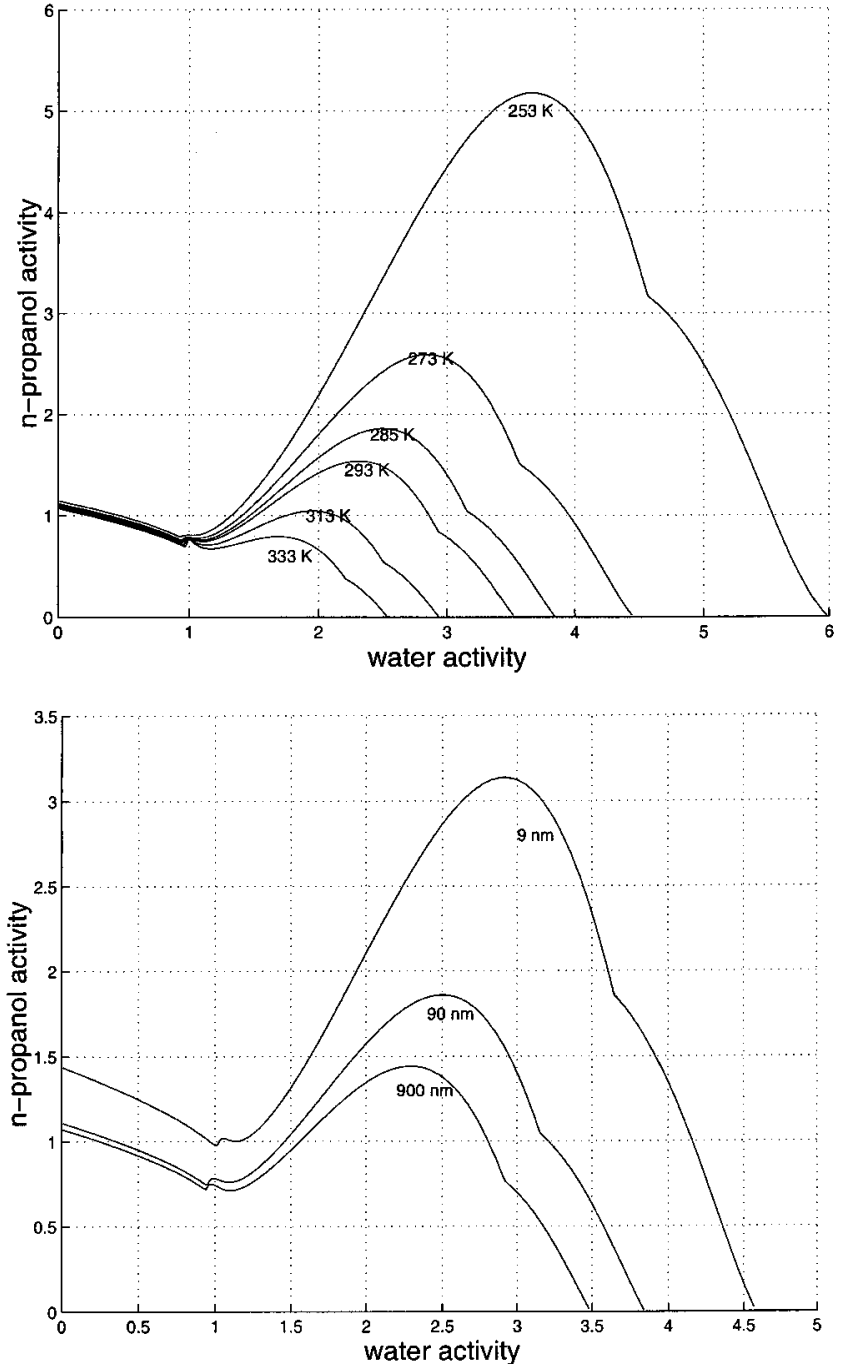

Figure 4. Modeled onset activities for water-n-propanol system (a) at different temperatures (mean diameter $90 \mathrm{~nm}$ ) and (b) with different substrate size distributions $(T=285 \mathrm{~K})$ using mole fraction dependent contact angle. ${ }^{31}$

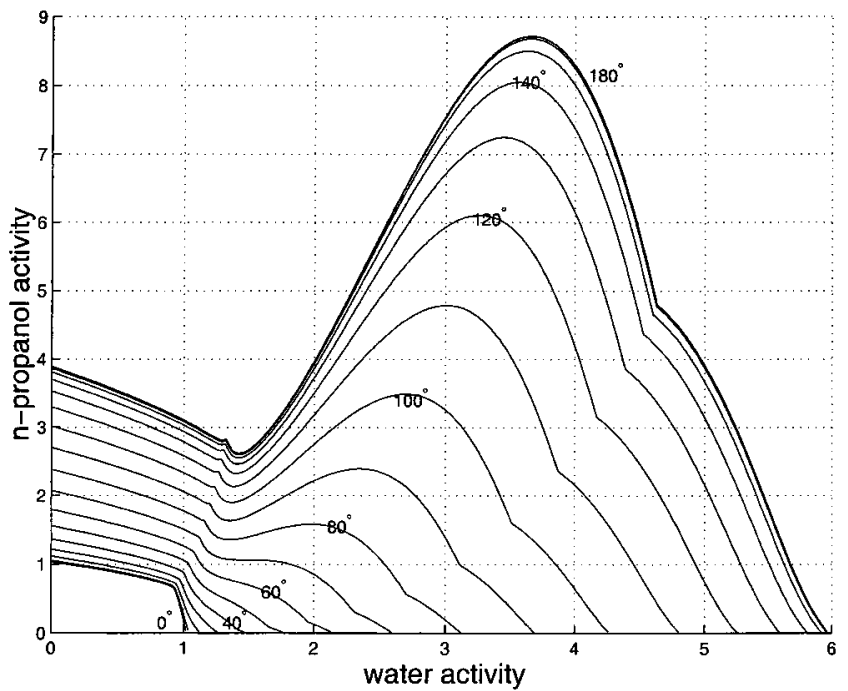

Figure 5. Modeled onset activities for water- $n$-propanol system for different values of constant contact angles. $T=285 \mathrm{~K}$, and the mean diameter is $90 \mathrm{~nm}$.

In Figure 10 the modeled onset values are compared with the experimental values presented in section 3. If we use constant 


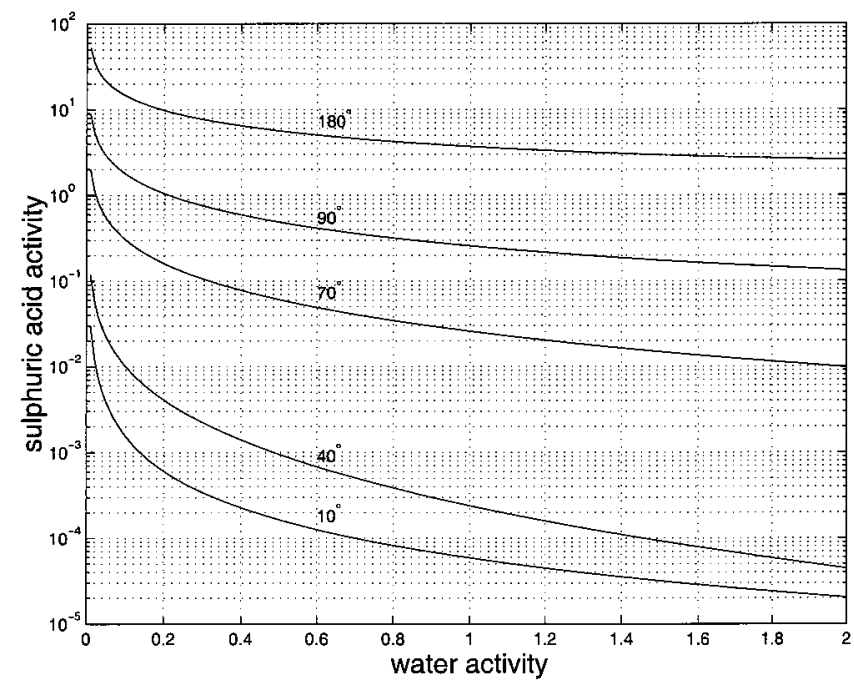

Figure 6. Modeled onset activities for water-sulfuric acid system different values of constant contact angles. $T=285 \mathrm{~K}$, and the mean diameter is $90 \mathrm{~nm}$.

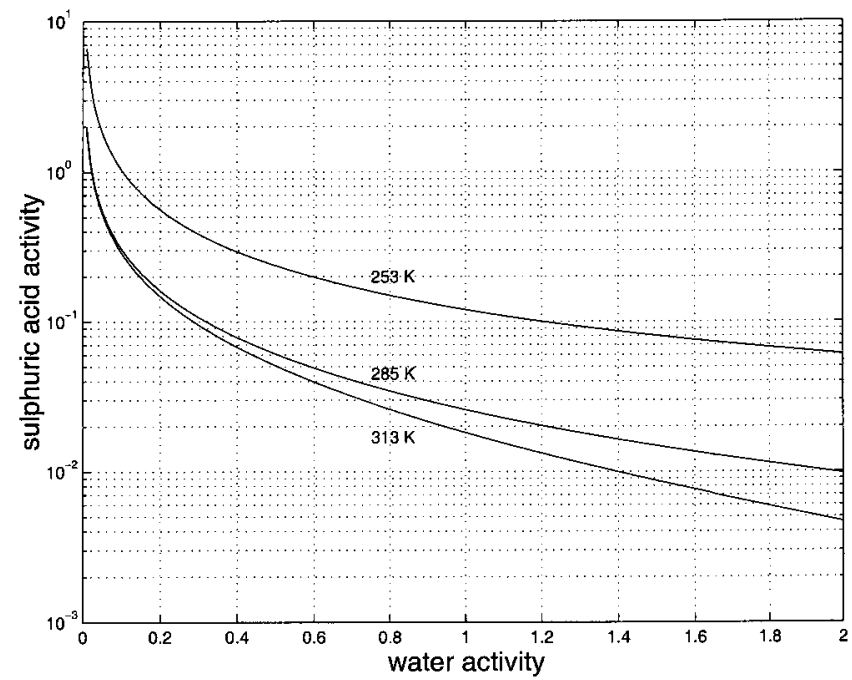

Figure 7. Modeled onset activities for water-sulfuric acid system at different temperatures. The contact angle is $70^{\circ}$, and the mean diameter is $90 \mathrm{~nm}$.

contact angle $\left(0^{\circ}\right)$ we do not see any hump nor spike. This agrees pretty well with the experimental results. However, if we use measured (varying) contact angle the difference between measured and modeled values is huge. Actually, the case of heterogeneous nucleation is even worse than the homogeneous one since at the water end there is no agreement between the theory and the experiments. In modeled onset values a small spike and a big hump can be seen. The contact angle changes very rapidly as water activity varies around 1 , as can be seen in Figure 11.

It is evident that the hump is of the same origin as the hump seen in theoretical activity curves of surface active mixtures in homogeneous nucleation. The hump represents completely unphysical behavior, since the nucleation rate goes down with increasing vapor pressure. The activity derivatives of the nucleation rate are related to number of molecules in the critical cluster through nucleation theorems. ${ }^{33-37}$ Oxtoby and Laaksonen $(1995)^{38}$ have shown that the hump corresponds to a negative number of molecules of one species in the critical cluster.

The underlying reason for the false behavior of the theory is that the assumptions made are conflicting: the equimolar surface does not coincide with the surface of tension for surface active

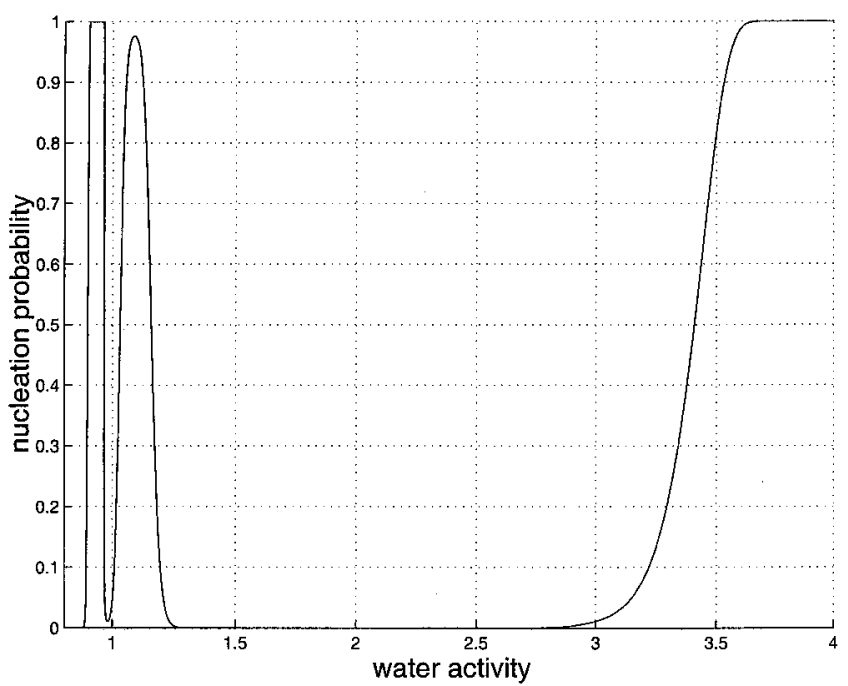

Figure 8. Nucleation probability as a function of water activity. The activity of $n$-propanol is 0.77 , the mean diameter is $90 \mathrm{~nm}$, and $T=$ $285 \mathrm{~K}$.

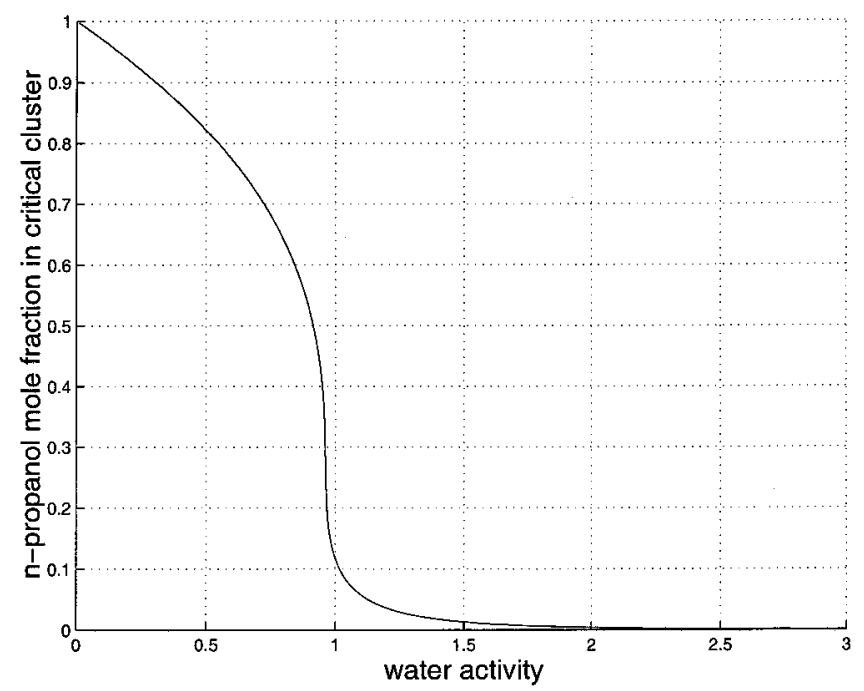

Figure 9. $n$-Propanol mole fraction in a critical cluster as a function of water activity. $T=285 \mathrm{~K}$, and $n$-propanol activity is 0.77 . Contact angle changes as a function of mole fraction.

systems, as shown by Laaksonen et al. (1999). ${ }^{21}$ Thus the capillarity approximation is not valid for these systems, and the surface tension of a curved surface cannot be taken to be that of a planar one. As shown in another paper in this issue, ${ }^{39}$ the capillarity approximation breaks down most severely for clusters which have a low surfactant (in this case propanol) concentration in the cluster interior, and a high concentration in the surface layer; such clusters occur just at vapor activities corresponding to the hump. The problem gets worse when the vapor activities are increased, and the critical cluster size is decreased, as the difference of the radii to the (real) surface of tension and to the (real) equimolar surface specified by eq 4 becomes a more and more significant fraction of $r^{*}$. In heterogeneous nucleation, the vapor activities are of course higher at higher contact angle values and at higher surface curvatures.

Unlike with the hump, the origins of the spike seem related specifically to the classical equations of heterogeneous nucleation. Pinpointing the exact reason for its occurrence would require a thermodynamic analysis outside the scope of this paper; however, it can be speculated that the various surface energies applied in the heterogeneous nucleation expressions probably are the cause of the spike. 


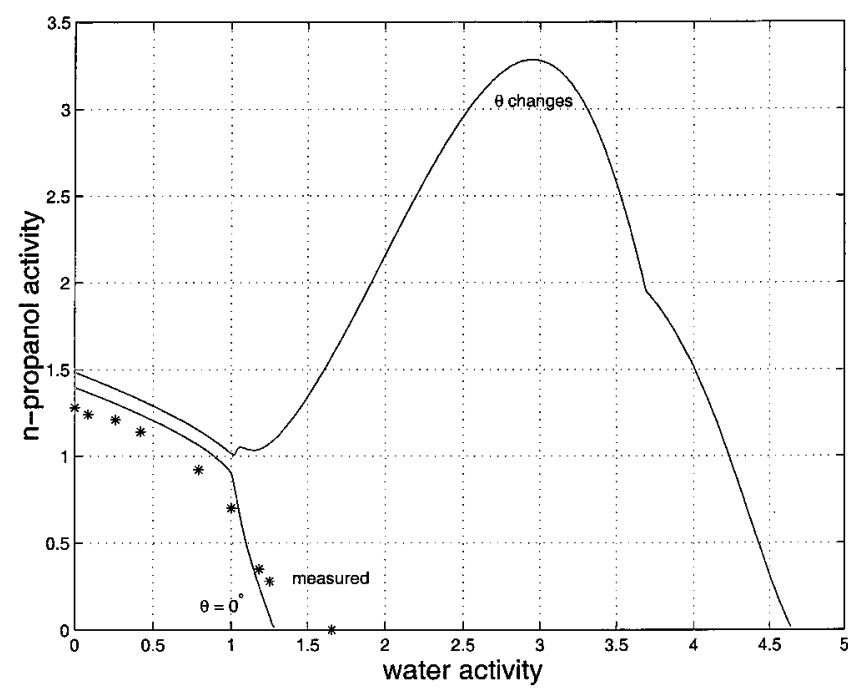

Figure 10. The experimental and modeled onset activities. Mean diameter is $8 \mathrm{~nm}$, and $T=285 \mathrm{~K}$.

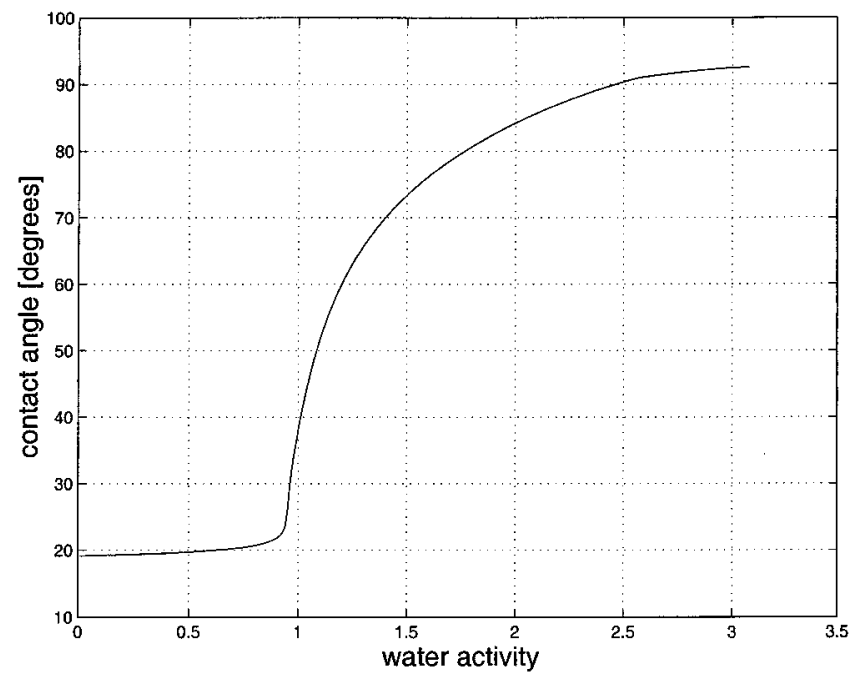

Figure 11. The critical cluster contact angle as a function of water activity in water $-n$-propanol system on onset activities based on experiments. $T=285 \mathrm{~K}$.

\section{Conclusions}

Using modeled onset activities for heterogeneous nucleation of water- $n$-propanol vapor on solid substrate a strange "hump" as well as a smaller "spike" can be seen clearly in the model results assuming a changing contact angle. However, both of these odd behaviors disappear, when the contact angle approaches zero (totally wettable surface). This kind of "hump" was first noticed in homogeneous nucleation by Garnier et al. $(1985)^{40}$ and in heterogeneous nucleation by Petersen et al. (1999). ${ }^{31}$ On the other hand, the quantitative experiments presented in section 3 do not show any humps nor spikes.

However, in the case of binary heterogeneous nucleation of water and sulfuric acid, neither "hump" nor "spike" can be seen in the modeled onset activities. This suggests that for a hump to appear a surface active substance is needed like it is the case in the water-alcohol systems. From our numerical results we can also see that the hump is decreasing with increasing temperature.

A major difficulty in calculating the onset activities lies in determining the contact angle between the parent aerosol particle and the newly formed embryo. For example, there are no reliable estimations of the contact angle of binary $\mathrm{H}_{2} \mathrm{SO}_{4}$ mixture. In some studies the angle is estimated to be approximately $70^{\circ} .^{26}$ However, the measurements of the contact angles of water$n$-propanol mixture show great variation of the contact angle in different compositions. ${ }^{31,23}$

\section{Appendix}

The thermodynamical parameters used in the model calculations were as follows.

The saturation vapor pressures for water, ${ }^{41} n$-propanol,${ }^{42}$ and sulfuric acid 25,43 are given by

$$
\begin{gathered}
p_{\mathrm{H}_{2} \mathrm{O}}=\exp \left(77.34491296-\frac{7235.424651}{T}-\right. \\
\left.8.2 \ln T+5.7113 \times 10^{-3} T\right) \\
p_{\mathrm{C}_{3} \mathrm{H}_{8} \mathrm{O}}=\exp \left(89.5883-\frac{8559.6064}{T}-9.29 \ln T\right) \\
p_{\mathrm{H}_{2} \mathrm{SO}_{4}}=\exp (-10156 a-0.414)
\end{gathered}
$$

where

$$
a=\frac{1}{T}-\frac{1}{T_{0}}+0.38 T_{\mathrm{r}}\left(\frac{1}{T}-\frac{1}{T_{0}}\right)+\frac{0.38}{T_{\mathrm{c}}\left(1-T_{\mathrm{r}}\right)} \ln \left(\frac{T}{T_{0}}\right)
$$

and

$$
T_{0}=360.15 \mathrm{~K}
$$

and

$$
T_{\mathrm{c}}=905.14 \mathrm{~K}
$$

and

$$
T_{\mathrm{r}}=\frac{T_{\mathrm{a}}}{T_{\mathrm{c}}}
$$

The calculations give the vapor pressures in $\left[\mathrm{N} \mathrm{m}^{-2}\right]$, all temperatures are in $\mathrm{K}$.

The densities of the binary mixtures of water and $n$-propanol ${ }^{44}$ and water and sulfuric acid ${ }^{45}$ were calculated by

$$
\begin{gathered}
\rho_{\mathrm{H}_{2} \mathrm{O}+\mathrm{C}_{3} \mathrm{H}_{8} \mathrm{O}}=X \rho_{\mathrm{p}}+(1-X) \rho_{\mathrm{w}} \\
\rho_{\mathrm{H}_{2} \mathrm{O}+\mathrm{H}_{2} \mathrm{SO}_{4}}=\rho_{1}+\frac{\left(\rho_{2}-\rho_{1}\right)(T-273.15)}{60}
\end{gathered}
$$

where $X$ corresponds to the mass fraction of $n$-propanol in the mixture, $\rho_{\mathrm{w}}$ is the density of water, and $\rho_{\mathrm{p}}$ is the density of $n$-propanol:

$$
\begin{gathered}
\rho_{\mathrm{w}}=\frac{w_{1}}{w_{2}} \\
\rho_{\mathrm{p}}=1047.94-0.835978 T
\end{gathered}
$$

where

$$
\begin{gathered}
w_{1}=999.83952+16.945176 T_{\mathrm{c}}-7.9870401 \times 10^{-3} T_{\mathrm{c}}{ }^{2}- \\
46.170461 \times 10^{-6} T_{\mathrm{c}}^{3}+105.56302 \times 10^{-9} T_{\mathrm{c}}{ }^{4}- \\
280.54253 \times 10^{-12} T_{\mathrm{c}}^{5} \\
w_{2}=1+16.87985 \times 10^{-3} T_{\mathrm{c}}
\end{gathered}
$$


where

$$
T_{\mathrm{c}}=T-273.15 \mathrm{~K}
$$

Parameters $\rho_{1}$ and $\rho_{2}$ are defined by

$$
\begin{gathered}
\rho_{1}=998.94+748.23 X-4.07622 X^{2}+317.88 X^{3} \\
\rho_{2}=982.99+608.19 X+233.26 X^{2}+154.19 X^{3}
\end{gathered}
$$

when $X<0.6$ and

$$
\begin{aligned}
& \rho_{1}=473.52+4903.99 X-11916.5 X^{2}+ \\
& 15057.6 X^{3}-6668.7 X^{4} \\
& \rho_{2}=250.52+5733.14 X-13138.14 X^{2}+ \\
& 15565.78 X^{3}-6618.37 X^{4}
\end{aligned}
$$

when $X \geq 0.6$.

All densities are in units $\left[\mathrm{kg} \mathrm{m}^{-3}\right]$ and temperatures in $\mathrm{K}$.

The surface tensions for water- $n$-propanol ${ }^{46}$ and watersulfuric acid ${ }^{47}$ liquid mixtures are given by

$$
\begin{aligned}
\sigma_{\mathrm{H}_{2} \mathrm{O}+\mathrm{C}_{3} \mathrm{H}_{8} \mathrm{O}} & =0.001 \exp \left(\sigma_{1}+\sigma_{2}+\sigma_{3}+\sigma_{4}+\sigma_{5}\right) \\
\sigma_{\mathrm{H}_{2} \mathrm{O}+\mathrm{H}_{2} \mathrm{SO}_{4}} & =0.001\left[\sigma_{6}+\frac{\left(\sigma_{7}-\sigma_{6}\right)(T-283.15)}{40}\right]
\end{aligned}
$$

where

$$
\begin{gathered}
\sigma_{1}=4.811191-1.951091 \times 10^{-3} T, \text { if } y<0.006 \\
\sigma_{1}=4.859191-1.951091 \times 10^{-3} T-8 y, \text { if } y \geq 0.006 \\
\sigma_{2}=(5.723187-0.03852713 T) y \\
\sigma_{3}=(-32.01079+0.1481755 T) y^{2} \\
\sigma_{4}=(45.29557-0.1891045 T) y^{3} \\
\sigma_{5}=(-19.68398+0.07811035 T) y^{4}
\end{gathered}
$$

where

$$
y=\frac{7 x}{1+6 x}
$$

and

$$
\begin{array}{r}
\sigma_{6}=74.00296+7.68634 x+625.86132 x^{2}- \\
5117.53488 x^{3}+10646.24244 x^{4}
\end{array}
$$

when $x<0.16$, and

$$
\sigma_{6}=77.04932+9.73321 x-59.4238 x^{2}+5.6594 x^{3}+
$$

when $x \geq 0.16$,

$$
\sigma_{7}=67.822+78.97377 x-207.81448^{2}-165.6474 x^{3}+
$$

when $x<0.25$, and

$$
\sigma_{7}=72.55489+32.99004 x-115.88314 x^{2}+62.0346 x^{3}
$$

when $x \geq 0.25$.

In the equations above, $x$ is the mole fraction of $n$-propanol or sulfuric acid in the mixture. All temperatures are in K.

The activity coefficients were determined for water $-n$ propanol solution using the parametrization of Viisanen et al. $(1998)^{48}$ and for water-sulfuric acid solution using the parametrization of Taleb et al. (1996). ${ }^{49}$

\section{References and Notes}

(1) Charlson, R. J.; Wigley, T. M. L. Sci. Am. 1994, 270, 48-57.

(2) Dockery, D. W.; Pope, C. A. Annu. Rev. Public Health 1994, 15 $107-132$.

(3) Laaksonen, A.; Talanquer, V.; Oxtoby, D. W. Annu. Rev. Phys. Chem. 1995, 46, 489-524.

(4) Arstila, H.; Laasonen, K.; Laaksonen, A. J. Chem. Phys. 1998, 108 , $1031-1039$

(5) Kusaka, I.; Wang, Z.-G.; Seinfeld, J. H. J. Chem. Phys. 1998, 108, $3416-3423$

(6) Seinfeld, J. H.; Pandis, S. N. Atmospheric Chemistry and Physics: From Air Pollution to Climate Change; John Wiley \& Sons: New York, 1998.

(7) Korhonen, P.; Kulmala, M.; Viisanen, Y. J. Aerosol Sci. 1997, 28 (6), 901-917.

(8) Clegg, S. L.; Brimblecombe, P.; Wexler, A. S. J. Phys. Chem. A 1998, 102, 2137-2154.

(9) Flood, H. Z. Phys. Chem. A 1934, 170, 286-295.

(10) Reiss, H. J. Chem. Phys. 1950, 18, 840-848.

(11) Doyle, G. J. J. Chem. Phys. 1961, 35, 795-799.

(12) Heist, R. H.; Reiss, H. J. Chem. Phys. 1974, 61, 573-581.

(13) Jaecker-Voirol, A.; Mirabel, P.; Reiss, H. J. Chem. Phys. 1987, 87, 4849-4852.

(14) Wilemski, G. J. Chem. Phys. 1984, 80, 1370-1372.

(15) Laaksonen, A.; Kulmala, M.; Wagner, P. E. J. Chem. Phys. 1993, 99 (9), 6832-6835.

(16) Kulmala, M.; Laaksonen, A.; Pirjola, L. J. Geophys. Res. 1998, $103,8301-8308$

(17) Fletcher, N. H. J. Chem. Phys. 1958, 29, 572

(18) Lazaridis, M.; Kulmala, M.; Laaksonen, A. B. J. Aerosol Sci. 1991 22,823

(19) Zapadinsky, E.; Gorbunov, B.; Voloshin, V.; Kulmala, M. J. Colloid Interface Sci. 1994, 166, 286-293.

(20) Kulmala, M.; Lazaridis, M.; Laaksonen, A.; Vesala, T. J. Chem. Phys. 1991, 94 (11), 7411-7413.

(21) Laaksonen, A.; McGraw, R.; Vehkamäki, H. J. Chem. Phys. 1999, $111,2019-2027$.

(22) Abraham, F. F. Homogeneous Nucleation Theory; Academic Press: New York, 1974

(23) Ortner, R. Experimentelle Bestimmungen von Kontaktwinkeln unärer und binärer Flüssigkeiten bezüglich verschiedener Substrate und Vergleich der Wilhelmy Methode mit dem goniometrischen Verfahren. Master's Thesis, Universität Wien, Vienna, 2000.

(24) Stauffer, D. J. Aerosol Sci. 1976, 7, 319-333.

(25) Kulmala, M.; Laaksonen, A. B J. Chem. Phys. 1990, 93 (1), 696701.

(26) Hamill, P.; Turco, R. P.; Kiang, C. S.; Toon, O. B.; Whitten, R. C. J. Aerosol Sci. 1982, 13, 561 .

(27) Adamson, A. W. Physical Chemistry of Surfaces, 4th ed.; Wiley: New York, 1982

(28) Lazaridis, M.; Kulmala, M.; Gorbunov, B. Z. J. Aerosol Sci. 1992, 23 (5), 457-466.

(29) Wagner, P. E. J. Colloid Interface Sci. 1985, 105, 456

(30) Vesala, T.; Kulmala, M.; Rudolf, R.; Vrtala, A.; Wagner, P. E.J. Aerosol Sci. 1997, 28 (4), 565-598.

(31) Petersen, D.; Ortner, R.; Vrtala, A.; Laaksonen, A.; Kulmala, M.; Wagner P. E. J. Aerosol Sci. 1999, 30, S35.

(32) Strey, R.; Wagner, P. E.; Viisanen, Y. Homogeneous Nucleation Rates of Particle Formation from Vapor Mixtures. In Nucleation and Atmospheric Aaerosols. Proceedings of the Thirteenth International Conference; Fukuta, N., Wagner, P. E., Eds.; A. Deepak Puslishing: Hampton, Virginia, 1992; pp 111-120.

(33) Kashchiev, D. J. Chem. Phys. 1982, 76, 5098-6102.

(34) Viisanen, Y.; Strey, R.; Reiss, H. J. Chem. Phys. 1993, 99, 46804692.

(35) Oxtoby, D. W.; Kashchiev, D. J. Chem. Phys. 1994, 100, 76657671. 
(36) Ford, I. J. J. Chem. Phys. 1996, 105, 8324-8332.

(37) Vehkamäki, H.; Ford, I. J. J. Chem. Phys. 2000, 113, 3261-3269.

(38) Oxtoby, D. W.; Laaksonen, A. J. Chem. Phys. 1995, 102, 68466850.

(39) Laaksonen, A.; Napari, I. J. Phys. Chem. B 2001, 105, 11678.

(40) Garnier, J. P.; Mirabel, P.; Migault, B. Chem. Phys. Lett. 1985, $115,101-103$.

(41) Preining, O.; Wagner, P. E.; Pohl, F. G.; Szymanski, W. Heterogeneous Nucleation and Droplet Growth; University of Vienna, Institute of Experimental Physics: Vienna, Austria, 1981.

(42) Schmeling, T.; Strey, R. Ber. Bunsen-Ges. Phys. Chem. 1983, 87, $871-874$
(43) Ayers, G. P.; Gillett, R. W.; Gras, J. L. Geophys. Res. Lett. 1980 , 7, 433-436

(44) Kell, G. S. J. Chem. Eng. Data 1975, 20, 97-105.

(45) Pruppacher, H. R.; Klett, J. D. Microphysics of Clouds and Precipitation; Reidel: Dordrecht, The Netherlands, 1978.

(46) Teitelbaum. Zh. Fiz. Khim. 1951, 25, 911-919.

(47) Sabinina, L.; Terpugow, L. Z. Phys. Chem. A 1935, 173, 237241.

(48) Viisanen, Y.; Wagner, P. E.; Strey, R. J. Chem. Phys. 1998, 108, 4257-4266.

(49) Taleb, D.-E.; Ponche, J.-L.; Mirabel, P. J. Geophys. Res. 1996, 101 25967-25977. 